\title{
Gut microbiome-derived lactate promotes to anxiety-like behaviors through GPR81 receptor- mediated lipid metabolism pathway
}

\section{Baixi Shan}

Jiangxi University of Traditional Chinese Medicine

\section{Yonggui Song}

Jiangxi University of Traditional Chinese Medicine

\section{Sufen Zeng}

Jiangxi University of Traditional Chinese Medicine

Jiagui Song

Peking University

\section{Qiang Zeng}

Jiangxi University of Traditional Chinese Medicine

\section{Zhou Liao}

Jiangxi University of Traditional Chinese Medicine

\section{Tingting Wang}

Jiangxi University of Traditional Chinese Medicine

\section{Chao Huang}

Jiangxi University of Traditional Chinese Medicine

Dan Su ( $\sim$ sud94@aliyun.com )

Jiangxi University of Traditional Chinese Medicine https://orcid.org/0000-0001-9610-8072

\section{Research}

Keywords: Gut microbiome, GPR81, Lactic acid, Anxiety, Fecal microbiota transplant, Metabonomics.

Posted Date: November 13th, 2019

DOI: https://doi.org/10.21203/rs.2.17202/v1

License: (c) (1) This work is licensed under a Creative Commons Attribution 4.0 International License. Read Full License 


\section{Abstract}

Accumulating evidence suggests that chronic stress can perturb the composition of the gut microbiota and induce host anxiety-like behavior. In particular, microorganism-derived products that can directly or indirectly signal to the nervous system. This study sought to investigate whether high levels of Lactobacillus and lactic acid in the gut of rats under chronic unpredictable stress were the factors leading to anxiety. Results: Oral lactic acid rat and transplantation of fecal microbiota from anxious rats into antibiotic-treated rat caused behavioral abnormalities such as psychomotor hyperactivity, impaired learning and memory in the recipient animals. These rats also showed inhibition of the adenylate cyclaseprotein kinase A pathway of lipolysis after activation of G protein-coupled receptor 81 (GPR81) by lactic acid in the liver, as well as increased TNF-a, compared with healthy controls. Furthermore, we show that sphingosine-1-phosphate receptor 2 (S1PR2) protein expression in hippocampus is reduced in chronic unpredictable stress compared to control subjects and its expression negatively correlates with symptom severity. Conclusions: our study suggests that the gut microbiome-derived lactate promotes to anxiety-like behaviors through GPR81 receptor-mediated lipid metabolism pathway.

\section{Background}

Chronic stress increases the risk of developing many psychiatric disorders such as anxiety, depression [1, 2]. Early studies show that stress can perturb the composition of the gut microbiota and that enteric pathogens can affect host behavior [3], such as immune and nervous system function, which is closely related to memory, cognition, locomotor activity, sleep quality, mood changes and circadian rhythms [4-8]. Variations in the composition of gut microbiota have been reported to have crucial roles in the pathogenesis of psychiatric diseases, including anxiety and schizophrenia, and differences in gut microbiota have been identified in humans with a variety of psychiatric diseases, which suggests that gut microbiota can influence brain function and behavior through the microbiota-gut-brain axis [9-12]. Later research has started to uncover gut-brain communication-in particular, microorganism-derived metabolites that can directly or indirectly signal to the nervous system $[3,13,14]$. However, it is not clear whether intestinal metabolites contribute to anxiety-like behavior. Initially, we screened gut microbiota and metabolites by $16 \mathrm{~S}$ rRNA sequencing and metabonomics. It is found that gut microbiota changed in chronic stress rats. Especially, there were more Lactobacillus in the gut of chronic stress rats compared with the control group.

Some literatures reported that Lactobacillus can cause a large accumulation of bacteria in the small intestine, and high levels of lactic acid were produced by Lactobacillus fermenting sugar in their food, which can further lead to brain fogginess [15] and memory, cognition impairment [16]. However, it is still unknown whether gut microbiome-derived lactate acts on anxiety. Furthermore, we systematically studied the role of gut microbiome-derived lactate in specific signaling pathways and mechanisms between gut and brain. And the effects on anxiety-like behaviors of lactic acid have been compared and evaluated. These may provide a reference for targeted treatment of anxiety. At the same time, it helps to make rational use and correct understanding of Lactobacillus. 
In our study, GPR81 lactic acid receptor was activated by lactic acid produced by gut microbial metabolism, inducing anxiety-like behavior by regulating lipolysis leading to disorder of lipid metabolism. What's more, lipids, such as sphingomyelin, play a very important role in reducing inflammation and promoting stress recovery. Therefore, we investigated the direct links between the gut microbiome and metabolome and their impact on anxiety, behavior in chronic unpredictable stress rats using the combination of microbiome and metabolome analyses. We found that lactobacillus-derived molecules was one of the regulators of emotional and cognition, targeting different organ-specific receptors throughout the body, and ultimately induce anxiety-like behavior and inflammatory processes.

\section{Results}

\section{Exposure to chronic unpredictable stress (CUS) leads to emotional, memory behavior impairments and altered cerebral blood flow}

The experimental design was shown as Fig. 1a. In studies with adult male rats, we used a paradigm in which rats exposed to different chronic stresses daily showed a passive response to different stressful environments (Fig. S1). These rats display increases in anxiety-like behaviors indicating vulnerability to the effects of chronic unpredictable stress environments. Compared with the control group, the rats in the chronic unpredictable stress group body weight gained significantly lower (Fig. 1b). After rats were exposed to chronic unpredictable stress conditions, behavioral tests such as open field test (Fig. 1c), forced swimming test (Fig. 1d), tail suspension test (Fig. 1e), elevated plus maze test (Fig. 1f) and tube test (Fig. 1g) were performed on those rats. As shown in Fig. 1j-m, open field test revealed that time spend in the center, frequency of center entries were significantly reduced in rats exposed to CUS conditions when compared to control group but no significant difference between distance moved and average speed. There was a significant effect of exposure to CUS on anxious like behavior, measured by a decreased time spent on the open-arms of the elevated plus maze test and an increased time spent in the closed arms by CUS group when compared to controls, no significant difference in time in the center (Fig. 1n-p). Forced swim test (Fig. 1q) and tail suspension test (Fig. 1r) also showed that immobility was increased in rats exposed to CUS conditions. In addition, there was a significant correlation between cerebral blood flow and the degree of cognitive impairment [29]. Chronic cerebral hypoperfusion is a direct cause of ischemic hypersensitive areas such as white matter and hippocampus [30]. Among them, ischemia and hypoxia occur in brain tissue under chronic perfusion insufficiency, resulting in disorder of emotional management function in patients' brain [31]. Under the combined action of many factors, such as environmental stimulus, , anxiety and other negative psychological emotions appear. As shown in Fig. $1 \mathrm{i}$ and Fig.1s, the cerebral blood flow of rats under chronic unpredictable pressure was significantly lower than that of the control group. These results suggest that CUS can induce anxiety-like behaviors in rats.

\section{Anxiety rats exhibit altered gut microbiota}


16S rRNA sequence analysis was performed to determine the composition of faeces microbiota of the study animals. In total, we obtained 509208 high-quality reads across all samples with an average number of 42434 reads per sample. These reads were clustered into 665 OTUs (operational taxonomic units) at $97 \%$ sequence similarity. The composition of gut microbiome changed in anxious rats (Table S1). A Venn diagram showed that 528 of 665 OTUs were detected in the two groups, while 73 and 64 OTUs were unique to patients with anxiety group and control group, respectively (Fig. 2e). Alpha diversity assesses differences in within-subjects diversity, alpha diversity was lower in anxiety rats relative to control rats. These data suggest that anxiety rat displayed decreasing separation in microbial principle components and decreasing difference in Shannon's diversity of microbiome (Fig. S2a). To determine whether the microbial composition of anxiety rat was substantially different from that of control group, we carried OUT $\beta$-diversity analysis (Fig. 2a) and found obvious differences in gut microbial composition between the two groups. Rarefaction curves tended to approach the saturation plateau, suggesting that the sequencing depth was enough to cover the whole bacterial diversity (Fig. 2b). The different abundant taxa between samples from control and anxiety rats are presented in Fig. S2b using phylum to genuslevel data. At the phylum level, the relative abundance showed that Firmicutes and Bacteroidetes were predominant in both groups. Firmicutes were the most dominant gut microbiota, accounting for an average of $59.05 \%$ and $53.58 \%$ of the sequences in the control and anxiety rats, respectively. Bacteroidetes were the second dominant gut microbiota, accounting for an average of $37.35 \%$ and $44.71 \%$ sequences in the control and anxiety rats, respectively (Fig. S2c). At the genus level, all the sequences could be assigned to 10 genus (Fig. 2c). Relative abundance of other microorganisms is shown in Fig. 2f. Bacteroidetes and Lachnospiraceae dominated the gut microbiome of both groups. Bacteroidetes were the most dominant gut microbiota, accounting for an average of $25.25 \%$ and $30.20 \%$ of the sequences in the control and anxiety rats, respectively. Lachnospiraceae were the second dominant gut microbiota, accounting for an average of $23.20 \%$ and $12.23 \%$ sequences in the control and anxiety rats, respectively (Fig. S2d). Wilcoxon rank-sum test bar plot on genus level indicate that anxiety group was no significant difference between Bacteroidetes and Lachnospiraceae but Lactobacillus $(P=0.02753)$, Alloprevotella $(P=0.02819)$, Prevotellaceae $(P=0.02819)$ and Corynebacterium $(P=0.02819)$ had significant difference compared with the control group (Fig. 2g). Among the four bacteria with significant differences, the relative abundance of Lactobacillus was the largest. This suggests that Lactobacillus, the third dominant gut microbiota, may be associated with anxiety disorder.

\section{Plasma metabolism are significantly altered in anxiety rats}

To assess the effects of the changes in the gut microbiota on metabolic pathways, we performed untargeted metabolome profiling to screen for major molecular differences using an orbitrap mass spectrometer (LTQ ORBITRAP VELOS PRO, Thermo Fisher Scientific, San Jose, CA, USA) equipped with a heated electrospray ionization (HESI) probe was coupled to the UHPLC system. Both positive and negative ion modes in MS were used to evaluate the plasma samples. Both ion mode were used for the final analysis and 5314 reproducible peaks including 5000 in positive ion mode (Fig. 3d) and 314 in 
negative ion mode (Fig. S3) were obtained. To evaluate the systemic changes of the metabolome in anxiety rats and find important metabolites, partial least-square discriminant analysis (PLS-DA) model was generated, which showed a clear separation between the anxiety and control groups. Fig. 3a shows separation of the two groups analyzed. The anxiety group is shown in blue, and the control group is shown in green. The OPLS-DA scores plot of control and anxiety rats showed good fit and high predictability of the model with values for $R^{2} Y$ (which assesses the extent to which the data fit the model) and the quality assessment metric $Q^{2}$ of 0.989 and 0.945 , respectively. We next looked for analytes with high variable importance in projection (VIP) scores, which assess the relative contribution to the OPLS-DA model. These analyses identified 130 metabolite (red circle) that showed a distinctly strong contribution relative to the other features, with a distinct VIP score $>1.5$. S-plots indicated the influence and contribution on anxiety rats compare to control rats (Fig. 3b). The metabolic classes were mainly grouped into lipids, including glycerophospholipids, glycerolipids, fatty acyls, sterols, and acyl carnitines. Compared to control rats, there were differences in plasma glycerophospholipids, lysophosphatide, acyl carnitines, fatty acyls, steroids in the anxiety group. Metabolite correlation analysis is shown in Fig. S4. The average relative concentration of plasma metabolites were significantly lower in the anxiety rats (Fig. $3 c)$. To assess the potential role of the altered metabolites in the pathogenesis of anxiety and, we performed a heatmap analysis of relative intensities of plasma metabolites in control and aniety rats (Fig. 3e). 44 metabolites were further selected based on fold changes (Anx/Con > 2.0 or Anx/Con < 0.50) and Mann-Whitney U tests. Plasma metabolites, including 2-linoleoyl-sn-glycero-3-phosphocholine, behenoylglycine, cholesterol sulfate, tsugaric acid, lysoPE (22:5) showed strong positive correlations with anxiety. The other 35 metabolites were all significantly reduced and showed negative correlations with anxiety in the rats. Most of the reduced metabolites belonged to glycerophospholipids, such as lysophosphatidylethanolamine, lysophosphatidylcholines, phosphatidylethanolamine, phosphatidylcholine, arachidonoylglycerophosphoinositol, triglycerides, monoacylglyceride, oleoylglycerophosphocholine (Fig. 3f). Significantly increased cholesterol sulfate, tsugaric acid B, behenoylglycine were observed in the anxiety rats compared to the control rats. Interestingly, the levels of sphingomyelin was significantly reduced in the anxiety rats, which was important substrates of relevance to stress-related psychiatric disorders. In addition, lactic acid levels was significantly increased in the anxiety rats. Most of the altered metabolic pathways were associated with the gut microbiota metabolism, which indicated the dysbiosis of the gut microbiota in the anxiety rats.

\section{Gut microbiome transplantation from anxiety rat induces anxiety-like behaviors in GF recipient rat}

To determine whether anxiety-like behavioral phenotypes might be linked with disturbed gut microbiota, we performed fecal microbiota transplantation experiments (Fig. 4a). This approach has been successfully used to determine the causative role of gut microbiota in the schizophrenia, anxiety-like behaviors and autism symptoms [20,32,33]. The global gut microbial phenotypes of the randomly selected subset samples used for these FMT experiments were representative of their full population distributions. We depleted the microbiota (Fig. 4b) in SPF male Sprague-Dawley rats by supplying a 
cocktail of antibiotics for 14 days, and then gavaged fecal phosphate buffer from anxious and control rat to antibiotic-treated rats (Fig. 4a). Initially, we measured the weight of the 0 day and 14 days of oral antibiotics and PBS experimental rats. There was no significant difference $(P=0.0635)$ in weight gain over the course of the experiment across groups (Fig. 4C), indicating no adverse effects of fecal transplants compared with gavage of PBS. These data show that oral gavage delivery of microbiota from anxiety fecal microbiome transplantation (AFMT) or control fecal microbiome transplantation (CFMT) rats effectively altered the microbiota of otherwise naive non-stressed rats (Fig. 4b). To test whether colonization of GF rat with ' microbiota' results in anxiety-like behaviors, four well-established behavioral tests-OFT, EPMT, FST and TST-were performed on post FMT. There were no significant differences in OFT, EPMT between 'microbiota' and 'healthy microbiota' recipient rat (Fig. S6). However, the duration of immobility in the forced swimming test was significantly increased in the AFMT rats compared to the vehicle rather than CFMT (Fig. 4e). In the TST, compared with vehicle and CFMT rats, the immobility time of AFMT rats increased significantly (Fig. 4f), suggesting increased anxiety-like (and more immobility) behavior. In addition, we performed cerebral blood flow experiments in rats transplanted with anxious microorganisms and control microorganisms. Interestingly, compared with rats in oral PBS group, rats transplanted with anxious microorganisms showed lower cerebral blood flow. However, there was no significant difference compared with the transplanted control microorganisms (Fig. 4d and Fig. 4r). In short, these behavioral tests showed that rat transplanted with anxiety microbiota displayed more immobility, increased anxiety-like behaviors, suggesting that the disturbed microbial composition of anxiety microbiota recipient rat was associated with several endophenotypes characteristic of rats models of anxiety.

\section{Anxiety rats were accompanied by high levels of lactic acid}

Gut microorganisms produce L-lactic acid and D-lactic acid. Effects of L-lactic acid and D-lactic acid on anxiety-like behavior were investigated. Initially, the concentrations of L-lactic acid and D-lactic acid in plasma and feces of rats were determined respectively. The results showed that the concentration of Llactic acid (Fig. 4i) and D-lactic acid (Fig. 4k) in plasma of rats in anxiety group significant increased, and concentration of L-lactic acid (Fig. 4n) in feces of rats in anxiety group significant increased. D-lactic acid in feces may be low in concentration or absorbed by the intestine but not detected. Compared to control group rat, the concentration of L-lactic acid (Fig. 4j) and D-lactic acid (Fig. 4I) in plasma of rats in oral administration of antibiotics significant reduced. Moreover, after transplantation of anxious microorganisms and control microorganisms into sterile rats, the levels of L-lactic acid and D-lactic acid in plasma of rats were measured. We found that the concentration of L-lactic acid (Fig. 40) and D-lactic acid (Fig. 4p) in plasma of transplanted anxious microorganisms and control microorganisms were higher than those of oral antibiotics group. Similarly, L-lactic acid in feces of microbial transplanted rats was also higher than that of oral antibiotics group (Fig. 4q), which indicates that lactic acid content in rats was closely related to gut microbiome. 
Both intense physical activity and potent psychosocial stressors increase blood lactate. Raising lactate levels by infusing the chemical can have an anxiogenic effect. To further verify that high levels of lactic acid in the blood affected emotional cognitive behavior and memory impairment, we still adopt classical behavioral testing methods-FST and TST-were performed on oral lactic acid rats. The experimental design as Fig. S7, we initially assessed oral L-lactic acid and D-lactic acid physiologically influences psychobehavioral characteristics by comparing with oral PBS rat. In the FST, immobility time is widely used as an index of anxiety-like behavior. Here, we found that oral L-lactic acid and D-lactic acid displayed increased anxiety-like behavior as evidenced by a significantly increased immobility time (Fig. 4g). Cognitive behaviors were also measured using TST. In the TST, there was no difference between the oral L-lactic acid groups and oral PBS group. The duration of immobility in the TST was significantly increased in the oral D-lactic acid rat compared to the oral PBS rat (Fig. 4h). In addition, cerebral blood flow was measured in oral lactic acid rats, which showed obvious cerebral ischemia compared with rats in oral PBS group (Fig. 4d and Fig. 4r).

\section{Perturbed lipid metabolism in anxiety microbiota recipient rat}

We further performed nontargeted lipid metabolomics to determine whether or which metabolisms modulated by the gut microbiome were paralleled by an altered microbiota-gut-brain axis. Based on the PLS-DA models of lipid metabolite profiling data, we found that the hippocampal lipid metabolites were significantly different between sterile rats transplanted with anxiety microorganisms and transplanted with healthy controls microorganisms (Fig. S5b). The rats transplanted with anxiety microorganisms status exhibited significantly different metabolite profiles compared with transplanted with healthy controls microorganisms. From the OPLS-DA models, we identified compounds that included 23 metabolites under lipid mode (Fig. S5a). We identified the differentially expressed metabolites between the two groups (Fig. S5d), and performed a heatmap analysis of relative intensities of lipid metabolomics in AFMT and CFMT rats (Fig. S5c). Of particular relevance to anxiety microbial transplantation, we measured differences in central nervous system and phospholipids metabolism, and in the anxiety microbiota compared to the healthy control microbiota recipient rats samples, we found abated sphingomyelin in the hippocampus, increased arachidonic acid and cholesterol in the hippocampus. The altered lipids were mainly glycerophospholipids including sphingomyelin (SM), phosphatidylethanolamines (PEs), lysophospholipid (LysoPC), cholesterol, dihydroceramide, cholesterol ester (CE) were generally decreased in the hippocampus of anxiety rat. The results for the anxiety microbiota rats suggested alterations in the glycerophosphatide metabolism. Taken together, the results suggested that the anxiety microbiota rats had significantly different lipid metabolite profiles compared with healthy controls, and the metabolite levels may further shift with different anxiety microbiota.

GPR81 was an G-protein-coupled receptor. Expression of GPR81 was highly restricted in the adipose tissues, suggesting that this orphan GPR may play a role in energy metabolism [34]. Many metabolites were recognized and bound by selective GPRs to elicit classical signal transduction events. These 
"signaling metabolites" include gut microbiota-derived products, such as lactate [35]. Lactic acid was an important metabolic intermediate released by skeletal muscle and other organs including the gut microbiome. The levels of lactic acid in plasma increase significantly following metabolic disorder of gut microorganisms or as a consequence of oxygen deficit [36], and this lactate elevations often links with a reduction of plasma free fatty acids [37]. Here we show that lactate activates the GPR81, which was expressed in liver and mediates antilipolytic effects through Gi-dependent inhibition of adenylyl cyclase.

The expression of the lactate receptor GPR81mRNA in liver, kidney and skeletal muscle, and its relative expression in liver is higher than that in other tissues [38]. By detecting the relative expression of GPR81 receptor in rat liver, we found that the expression of GPR81 increased significantly in anxious rats (Fig. 5d). Similarly, we examined the expression of GPR81 in the liver of oral L-lactic acid and D-lactic acid rats. The results showed that compared with PBS rats, the expression of GPR81 in liver of D-lactate rats increased, but there was no significant difference between oral L-lactate rats and PBS rats, and the expression of GPR81 in liver of transplanted anxious microorganism rats was significantly higher than that of oral antibiotic rats (Fig. 5e)

AC (Adenylyl Cyclase) was a key enzyme in the synthesis of cAMP (Cyclic Adenosine 3',5'-

monophosphate) from ATP, Gi was the dependent inhibition of adenylyl cyclase, and insufficient of cAMP has been linked to the downregulation of AC5. CAMP expression was reduced after AC5 inhibited by GPR81 receptor activated by lactate. and decreased expression of PKA due to insufficient cAMP, thereby acting as an anti-lipolysis. We evaluated the potential inhibitory effects of GPR81 recipient on adenylate cyclase 5 to reveal whether increased lactic acid contributed to reduced plasma free fatty acids and the AC5/PKA pathways. As shown in Fig. $5 \mathrm{a}$ and Fig. $5 f$, the anxiety rats showed a marked downregulation of AC5 and PKA protein expression. In addition, the expression of AC (Fig. 5g) and PKA (Fig. 5h) protein was significantly decreased in oral D-lactic acid rats, indicating inhibition of AC5/PKA signaling. Although there was a decrease in AC protein in oral L-lactic acid rats, but there was no significant difference. To verify whether lactic acid produced by intestinal microbial metabolism is involved in this signaling pathway, expression of AC5 (Fig. 5g) and PKA (Fig. 5h) proteins in AFMT, CFMT and oral antibiotictreated rats was detected. Interestingly, AFMT showed a stronger effect on these protein expression levels than oral antibiotic-treated rats, indicates that the gut microbiome is involved in the regulation of fatty acid metabolism.

In addition, we show that the S1PR2 in the medial prefrontal cortex of rats regulates resilience to chronic social defeat stress. S1PR2 expression was elevated in the medial prefrontal cortex of anxiety rat compared to control rats. Characterized by increased anxiety-like behaviors, and these effects were mediated by tumor necrosis factor (TNFa). Western blot analysis (Fig. $5 \mathrm{c}$ ) and immunofluorescence staining (Fig. 5b) showed that stress attenuated the pro-inflammation mediators S1PR2 expression, and increased expression of the TNFa (Fig. 5i). Furthermore, we found that sphingomyelin in plasma and brain was reduced in anxiety rat compared to control subjects and its expression negatively correlates with symptom severity. 


\section{Discussion}

The microbiome plays a key role in the brain development and behavior and participates in the pathophysiology of some major psychiatric disorders including anxiety [20]. In this study, we demonstrate that the chronic unpredictable stress induces anxiety-like behavior and cerebral ischemia in rat and profound (Fig. 1) gut microbiota alterations in anxiety rat visa-vis healthy control individuals (Fig. 2). We found that the microbial composition of rat with CUS was less diverse and associated with lower alpha diversity scores than that of healthy control individuals. Generally speaking, a high diversity is beneficial to health and the lower diversity suggests an overall abnormal microbial status within anxiety rat [39]. The gut microbiome of the anxiety rats was clearly different from that of the control rats. Interestingly, we found that rats with CUS had more Lactobacillus (Fig. 2c). Inconsistent with low levels of lactic acid bacteria in the gut of previously reported depressed patients [40]. This phenomenon may mean that too much Lactobacillus has a negative effect on the body's emotional cognitive behavior.

We generated GF rat whose guts were re-colonized with the fecal microbiota of anxiety rat or healthy controls, and found that a portion of the donor's microbiota established itself in the rat's guts via fecal transplantation (Fig. 4b). More notably, this alteration was transmissible: colonization of GF rat with 'microbiota' resulted in increased anxiety-like behaviors as compared with colonization with 'healthy microbiota' (Fig. 4e-f). Our animal experiments of GF rat colonization with microbiota resulted in anxietyrelevant behavioral changes similar to those observed in chronic unpredictable stress rat models of anxiety. We identified unique bacterial taxa and dysregulation of metabolites that were strongly associated with and anxiety severity (Fig. 2 and Fig. 3). These results highlight the role of gut microbiota as a potential causative factor in through their impact on host metabolism. In addition, we found profound alterations in the fatty acid metabolism pathway and inflammatory factors levels.

The functional analysis of the metabolomics dataset showed that the changes in the gut microbiota and dysregulation of plasma metabolites pointed to an association of the gut microbial dysbiosis with altered metabolisms of glycerophospholipids, glycerolipids, fatty acyls, sterols. Several metabolites, including sphingomyelin, lactic acid and other phospholipids have been identified and reported in anxiety rats [41]. The plasma levels of these metabolites reflect the balance between the rates of their generation and elimination. Our findings support the possibility that alterations of gut microbiota may potentially participate in the onset and/or pathology of anxiety and behavior through modulating fatty acid metabolic pathways.

Here, the anxiety-type behaviors was induced by the production of lactic acid metabolites in the gut from bacterial fermentation of carbohydrate substrates. Compared to L-isomer, D-lactic acid exhibted more anxiety behavior. We treated rats with antibiotic and then transferred 'anxiety microorganisms' to rats. These measures led to significant aggravate anxiety-type behaviors in $80 \%$ of individual and high levels of D-lactic acid were detected in plasma (Fig. 4p). Reaffirming that the symptoms were related to D-lactic acidosis. Likewise, the anxiety-type behaviors and cerebral ischemia of the rat with D-lactic acid group also deteriorated to a similar extent. The L-isomer of lactic acid is the main form of lactic acid in the 
human body and is produced by the enzymatic (L-lactate dehydrogenase) reduction of pyruvate. Dlactate is produced in much smaller quantities by the enzyme D-2-hydroxyl acid dehydrogenase that also metabolizes D-lactate in the human liver [42]. Unlike L-lactate that is efficiently metabolized, the breakdown of D-lactate via the methylglyoxal pathway is slow and limited which may contribute to the adverse effects observed with accumulation [43].

Liver is the most active site for lipid synthesis and the expression of GPR81 mRNA was also found in the liver [44]. In most cells, activation of Gi-type $G$ proteins results in an inhibition of adenylyl cyclase, and reduction of intracellular CAMP and thus protein kinase $A$ activity, and in turn the reduced phosphorylation of the lipid droplet coat protein perilipin and hormone sensitive lipase, which are key components of the TG hydrolytic system that generates FFA [45-47]. GPR81 is Gi coupled is consistent with the fact that infusion of lactate into humans can reduce plasma FFA. This is proposed to occur in a manner similar to the mechanism by which niacin lowers plasma FFA-specifically [34]. The finding that anxiety rats reduced S1PR2 in blood and brain suggests that S1PR2 may be a useful blood-based biomarker of resilience/vulnerability to stress. We demonstrated that chronic unpredictable stress increased TNFa in the hippocampus. As a whole, CUS leads to dysbacteriosis of the gut microbiome, and lactic acid, a metabolite of the flora, reduces plasma free fatty acid levels, such as sphingomyelin, by inhibiting fatty acid breakdown, and S1PR2 reduce stress-induced increases in inflammatory cytokines, specifically TNFa. More broadly, the results presented here highlight potential role of the gut microbiota in regulating for neuronal sphingolipids and their S1PR2 in mediating phenotypes important in stressrelated psychiatric diseases.

\section{Conclusions}

We have clearly shown that a chronic unpredictable stress rat cause anxiety-like behavior and aberrant structure of the gut microbiota and reduced plasma free fatty acid levels, demonstrating a contribution of gut dysbiosis to fatty acid metabolism and cognitive behavior in rat. Mechanistically, lactic acid metabolized by gut microbiota was involved in fatty acid metabolism and inflammation process in the body through GPR81. More broadly, the results presented here highlight potential regulatory roles for gut microbiota metabolite lactic acid and neuronal sphingolipids in mediating phenotypes important in stress-related psychiatric diseases. In particular, the finding that gut microbiota metabolite lactic acid correlated positively with anxiety symptom, and sphingolipids correlated negatively with anxiety symptom may provide the groundwork for the development of treatment strategies targeting sphingolipid receptors for stress-related psychiatric disorders including anxiety disorders.

\section{Methods And Materials}

\section{Animal}

Male Sprague-Dawley rats (180-220 g) were used in the animal experiments. Animal care was in accordance with the Guidelines for Animal Experimentation of Jiangxi University of Traditional Chinese 
Medicine (Nanchang, China) and the protocol was approved by the Animal Ethics Committee of the institution. Rats were bred in the Laboratory Animal Science and Technology Center, Jiangxi University of Traditional Chinese Medicine. They were kept in aseptic packing box until the start of experiments. Rats were fed autoclaved chow and water ad libitum under a 12-hour light/12-hour dark cycle (lights on at 7:30 a.m.) and constant temperature $\left(21^{\circ} \mathrm{C}\right.$ to $\left.22^{\circ} \mathrm{C}\right)$ and humidity $(55 \pm 5 \%)$ in barrier environment.

\section{Experimental design}

Rats were randomly assigned to either a anxiety group or a control group. The chronic unpredictable stress procedure was performed as described previously [17-19] with minor modification as indicated in Table 1. Rats in the control group were kept undisturbed in their home cages, while rats in the CUS group were exposed to various stress conditions for 30 days. Body weight was monitored every 5 days. Fresh fecal samples were collected on day 30 . After the completion of the behavioral test, all samples were collected at approximately $10 \mathrm{~h}$, frozen in liquid nitrogen stored at $-80^{\circ} \mathrm{C}$. Then, samples were processed for $16 \mathrm{~S}$ rRNA sequence, untargeted metabolomic, histological, PCR and western blot analyses.

\section{Behavioral testing}

Open-field test (OFT): Rats were placed individually in the corner of an open-field box $(\mathrm{L} \times \mathrm{W} \times \mathrm{H}, 100 \mathrm{~cm}$ by $100 \mathrm{~cm}$ by $40 \mathrm{~cm}$ ) and allowed to explore freely for $6 \mathrm{~min}$. Their spontaneous activities over the last 5 min were recorded. The total move distance, average moving speed, entry center frequency, spend time in the center were designated as an index of locomotor activity, while increased time, frequency or distance spent in the center indexes decreased anxiety. After testing each animal, the apparatus was cleaned with ethanol to remove olfactory cues.

Tail suspension test (TST): Rats were individually suspended by their tails using adhesive tape (distance from tip of tail was $2 \mathrm{~cm}$ ). Test sessions lasted for 6 min with the last 5 min scored for immobility. Rats that climbed on their tails were removed from further testing. Animals were considered to be immobile when they exhibited no body movement and hung passively [20]. Forced swimming test (FST): Individual rats were placed softly into Plexiglas cylinder (30 cm in height $\times 18 \mathrm{~cm}$ in diameter) filled with $30 \mathrm{~cm}$ water $\left(24 \pm 1^{\circ} \mathrm{C}\right)$. Rat's immobility and struggling behavior during the $5 \mathrm{~min}$ swim session were recorded and quantified by the investigators who were unaware of group assignment. Struggling was defined as vigorous movements of the forepaws breaking the water and immobility was defined when rat floated without struggling and making only those movements necessary to keep its head above the water [21].

Elevated plus maze test (EPMT): EPMT was a usual assessment for evaluating the anxiolytic-like effects. The apparatus was $100 \mathrm{~cm}$ above the ground including: two closed arms with dark walls $(60 \times 12 \times 40$ $\mathrm{cm})$ and two open arms $(60 \times 12 \mathrm{~cm})$. The arms were connected by the central platform $(12 \times 12 \mathrm{~cm})$. Rats were placed in the center area facing one of the closed arms, and then allowed to explore the maze 
for 5 min and defined as entering an arm when four paws crossed the dividing line. Time and entries into the open arms were considered as the anxiolytic indices by observers who were blind to the grouping. After each test, the maze was cleaned with ethanol to remove olfactory cues [22].

Tube test (TT): Tube test was a very interesting experiment. Two groups of rats were randomly placed on both sides of the test tube. Push-and-shove competition occurs when rats meet in tube. The number of losers was recorded as an anxiety index by the experimenters [23].

\section{Microbial DNA extraction and PCR amplification}

Microbial DNA was extracted from fecal samples using the OMEGA-soil DNA Kit (Omega Bio-Tek, U.S.) according to manufacturer's protocols. The final DNA concentration and purification were determined by NanoDrop 2000 UV-vis spectrophotometer (Thermo Scientific, Wilmington, USA), and DNA quality was checked by $1 \%$ agarose gel electrophoresis. The V3-V4 hypervariable regions of the bacteria 16S rRNA gene were amplified with primers 338F (5'- ACTCCTACGGGAGGCAGCAG-3') and 806R (5'-

GGACTACHVGGGTWTCTAAT-3') by thermocycler PCR system (GeneAmp 9700, ABI, USA). The PCR reactions were conducted using the following program: 3 min of denaturation at $95^{\circ} \mathrm{C}, 27$ cycles of $30 \mathrm{~s}$ at $95^{\circ} \mathrm{C}, 30 \mathrm{~s}$ for annealing at $55^{\circ} \mathrm{C}$, and $45 \mathrm{~s}$ for elongation at $72{ }^{\circ} \mathrm{C}$, and a final extension at $72{ }^{\circ} \mathrm{C}$ for 10 min. PCR reactions were performed in triplicate $20 \mu \mathrm{L}$ mixture containing $4 \mu \mathrm{L}$ of $5 \times$ FastPfu Buffer, $2 \mu \mathrm{L}$ of $2.5 \mathrm{mM}$ dNTPs, $0.8 \mu \mathrm{L}$ of each primer $(5 \mu \mathrm{M}), 0.4 \mu \mathrm{L}$ of FastPfu Polymerase and $10 \mathrm{ng}$ of template DNA. The resulted PCR products were extracted from a $2 \%$ agarose gel and further purified using the AxyPrep DNA Gel Extraction Kit (Axygen Biosciences, Union City, CA, USA) and quantified using QuantiFluor ${ }^{\text {TM}}$-ST (Promega, USA) according to the manufacturer's protocol.

\section{Processing of 16 S rRNA gene sequences}

Purified amplicons were pooled in equimolar and paired-end sequenced $(2 \times 300)$ on an Illumina MiSeq platform. Raw fastq files were demultiplexed, quality-filtered by Trimmomatic and merged by FLASH with the following criteria: (i) The reads were truncated at any site receiving an average quality score $<20$ over a 50 bp sliding window. (ii) Primers were exactly matched allowing 2 nucleotide mismatching, and reads containing ambiguous bases were removed. (iii) Sequences whose overlap longer than 10 bp were merged according to their overlap sequence. Operational taxonomic units (OTUs) were clustered with $97 \%$ similarity cutoff using UPARSE (version $7.1 \mathrm{http}$ ///drive5.com/uparse/) and chimeric sequences were identified and removed using UCHIME. The taxonomy of each 16S rRNA gene sequence was analyzed by RDP Classifier algorithm (http://rdp.cme.msu.edu/) against the Silva (128) 16S rRNA database using confidence threshold of $70 \%$. These taxonomies were used to construct summaries of the taxonomic distributions of OTUs, which can then be applied to calculate the relative abundances of microbiota at 
different levels. Distance matrices (Beta diversity) between samples were generated on the basis of weighted (Bray-Curtis similarity) reported according to principal coordinate analysis (PCoA).

\section{Untargeted metabolomics and measurement of metabolites}

The plasma samples and brain tissue samples were analyzed respectively by a $2.1 \times 100 \mathrm{~mm}$ ACQUITYTM $1.7 \mu \mathrm{m} \mathrm{C18}$ and $2.1 \times 100 \mathrm{~mm}$ ACQUITY ${ }^{\mathrm{TM}} 1.8 \mu \mathrm{m}$ HSS T3 using an orbitrap mass spectrometer (LTQ ORBITRAP VELOS PRO, Thermo Fisher Scientific, San Jose, CA, USA) equipped with a heated electrospray ionization (HESI) probe was coupled to the UHPLC system. The metabolomic procedure including sample preparation, metabolite separation and detection, data preprocessing and statistical analysis for metabolite identification was performed following previous protocols with minor modifications [24-26]. The methods of chromatographic separation and mass spectrometry were described in detail as follow: The UPLC analysis was performed with an UltiMate 3000 UHPLC system (Thermo Scientific, San Jose, CA, USA) equipped with a LTQ ORBITRAP VELOS PRO (Thermo Fisher Scientific, San Jose, CA, USA). Chromatographic separation was carried out at $40{ }^{\circ} \mathrm{C}$ on an ACQUITYTM UPLC C18 column $(2.1 \times 100 \mathrm{~mm}$, $1.7 \mu \mathrm{m}, \mathrm{UK})$ and an ACQUITYTM UPLC HSS T3 column $(2.1 \times 100 \mathrm{~mm}, 1.8 \mu \mathrm{m}$, UK). For plasma sample: the mobile phase consisted of water $(A)$ and methanol $(B)$, each containing $0.1 \%$ formic acid. The optimized UPLC elution conditions were: 0 3.0 min, 5.0\% 5.0\% B; $3.0 \sim 7.0 \mathrm{~min}, 5.0 \sim 54.0 \%$ B; $7.0 \sim 11.0 \mathrm{~min}$, 54.0 77.0\% B; 11.0 33.0 min, 77.0 100.0\% B; 33.0 38.0 min, 100.0\% B; 38.0 39.0 min, 100.0 5.0\% B; 39.0 44.0 min, 5.0\% B. For brain tissue sample: the mobile phase consisted of water $(A)$ and acetonitrile (B), each containing $0.1 \%$ formic acid. The optimized UPLC elution conditions were: $0 \sim 3.0 \mathrm{~min}$, 10.0\% 60.0\% B; 3.0 10.0 min, 60.0 80.0\% B; 10.0 16.0 min, 80.0 90.0\% B; 16.0 18.0 min, 90.0 100.0\% B; 18.0 20.0 min, 100.0\% B; $20.0 \sim 20.1 \mathrm{~min}, 100.0 \sim 10.0 \%$ B; $20.1 \sim 23.0 \mathrm{~min}, 10.0 \%$ B, The flow rate was $0.3 \mathrm{ml} / \mathrm{min}$. The autosampler was maintained at $4{ }^{\circ} \mathrm{C}$. Every $2 \mu \mathrm{l}$ sample solution was injected for each run. Mass spectrometry was performed on a LTQ ORBITRAP VELOS PRO (Thermo Fisher Scientific, San Jose, CA, USA). The scan range was from 100 to 1200 m/z. For HESI source, positive ion mode, Heater Temp, $350^{\circ} \mathrm{C}$; Capillary Temp, $320^{\circ} \mathrm{C}$, Sheath Gas Flow Rate, 35 arb; Aux Gas Flow Rate, 10 arb; I Spray Voltage, 4 kV; Capillary Voltage, 35 V; Tube Lens Voltage, $110 \mathrm{~V}$; sample first full scan, resolution of 30000. Data dependent scan (DDS) was used to select the top three peaks of the first abundance for CID fragment scanning and dynode detection. For HESI source, negative ion mode, Heater Temp, $300{ }^{\circ} \mathrm{C}$; Capillary Temp, $320^{\circ} \mathrm{C}$, Sheath Gas Flow Rate, 35 arb; Aux Gas Flow Rate, 10 arb; I Spray Voltage, $3.6 \mathrm{kV}$; Capillary Voltage, $35 \mathrm{~V}$; Tube Lens Voltage, $110 \mathrm{~V}$; sample first full scan, resolution of 30000. Data dependent scan (DDS) was used to select the top six peaks of the first abundance for CID fragment scanning and dynode detection. Dynamic exclusion was set to exclude a precursor ion for repeated MS/MS analysis within $15 \mathrm{~s}$. The activation type was collision induced dissociation (CID) and the intensity threshold was set at 1000 . All the acquisition and extraction of data were controlled by Thermo Xcalibur Roadmap software and SIEVE × 64 2.1.377 RELEASE. Then, the peak list was imported into SIMCA-P 14.0 software (Umetrics AB, Umea, Sweden) to acquire clustering information and important variables between the CUSP groups and the control group. Metabolites selected as biomarker 
candidates for further statistical analysis were identified on the basis of variable importance in the projection (VIP) threshold of 3 from the tenfold cross-validated PLS-DA model. The online HMDB database (http://www.hmdb.ca) (version: 4.0), KEGG database (http://www.genome.jp/kegg/) and Lipid maps Structure Database (LMSD) were used to align the molecular mass data $(\mathrm{m} / \mathrm{z})$ to identify metabolites. MetaboAnalyst (https://www.metaboanalyst.ca) (version 4.0) was used for the identification of metabolic pathways.

\section{Fecal microbiota transplantation experiments (FMT)}

To obtain samples for microbiota transplantation, fecal samples were collected $1 \mathrm{~h}$ after 30 days of chronic unpredictable stress. Then on days $1-14$, rats were fed freely with water containing antibiotics as Germ-free (GF) and record the weight. Another group of rats was administered normal water daily. Fecal samples from randomly chosen anxiety group and control group were used to colonize the guts of GF rat. The procedures of preparing the fecal samples for microbiota transplantation were as described in a previous study [27]. Fecal samples were freshly collected from adult SPF rats and homogenized in prereduced PBS at $2 \mathrm{~mL}$ per pellet. $1 \mathrm{~mL}$ of the settled suspension was administered by oral gavage to recipient GF rats. For mock treatment, rats were gavaged with pre-reduced PBS. The anxiety and control microflora receptor rats were cultured in a single cage in a sterile environment to prevent the normalization of intestinal flora. The weights of the rats were measured on day 1 and day 15 of FMT experiment. The behavioral tests (including FST and TST) were performed on 15 day after microbiota transplantation. Tissue samples were collected at the time the rats were killed and immediately snapfrozen in liquid nitrogen and stored at $-80^{\circ} \mathrm{C}$.

\section{Antibiotic treatment}

Antibiotic cocktails in drinking water containing, $50 \mathrm{mg} / \mathrm{ml}$ streptomycin, $100 \mathrm{mg} / \mathrm{ml}$ ampicillin, 50 $\mathrm{mg} / \mathrm{ml}$ vancomycin, $100 \mathrm{mg} / \mathrm{ml}$ metronidazole, $125 \mathrm{mg} / \mathrm{ml}$ ciprofloxacin, and $100 \mathrm{mg} / \mathrm{ml}$ ceftazidime were freshly prepared every two days and were given ad libitum for 2 week to rats. This protocol was chosen since it was reported not to promote loss of body weight $[27,28]$.

\section{Protein analysis}

Protein expression was analyzed by western blot analysis. Protein concentration was measured by Pierce $^{\mathrm{TM}}$ BCA Protein Assay Kit (23227, Thermo Scientific, USA). Liver tissues were homogenized in RIPA buffer with protease and phosphatase inhibitors. The protein extracts were separated by SDS-PAGE electrophoresis and transferred to a PVDF membrane. The membrane was incubated with antibodies against ADCY5/6 (Affinity, Cat\# DF3508), PKA (Affinity, Cat\# AF7746), the secondary antibodies of goat 
anti-rabbit (ZSGB-BIO, Cat\# ZB-2301), The membrane was then visualized by enhanced chemiluminescence western blotting detection reagent and protein concentrations were normalized by GAPDH expression. Shooting and analysis of signal intensities from immunoblots using AnalytikJena VisionWorks systems.

\section{Quantitative real-time PCR analysis}

Rat liver tissue was frozen in liquid nitrogen and stored at $-80^{\circ} \mathrm{C}$. Extraction of total RNA from frozen tissue with Trizol reagent. cDNA was synthesized from $1 \mu \mathrm{g}$ of total RNA with a Reverse Transcription Kit (TRANS, Cat\# AE311). The real-time PCR primer sequences are summarized in Supplementary Table 2.

\section{Immunofluorescence}

The following primary antibodies were used: rabbit anti-S1PR2 (Proteintech, Cat\# 21180-1-AP, 1:100), rabbit anti-TNF-a (Proteintech, Cat\# 17590-1-AP, 1:500). Paraffin-embedded rat brain sections (5- $\mu \mathrm{m}$ thickness) were prepared as a routine procedure. The sections were deparaffinized by three xylene washes, hydrated by alcohol and washed with distilled water. Antigen retrieval was performed by a microwave oven for $15 \mathrm{~min}$ in the citrate buffer $(10 \mathrm{mM}, \mathrm{pH}$ 6.0). The sections were added with $3 \%$ hydrogen peroxide to blockade endogenous peroxidase activity. 5\% BSA were incubated for $1 \mathrm{~h}$ at room temperature. Next, the sections were incubated with the primary antibody at $4{ }^{\circ} \mathrm{C}$ overnight. After being washed, the sections were incubated with the secondary antibody (FITC) at $37^{\circ} \mathrm{C}$ for $45 \mathrm{~min}$. DAPI were added to visualization of nuclei, then the sections were mounted by antifluorescence quencher. Shooting and analysis of slices using Leica TCS STED CW systems.

\section{Laser Doppler imaging of brain}

Laser Doppler images were acquired after CUSP using a laser Doppler imaging system (moorLDI2-HIR, Moor Instruments, DE, UK). During the procedure, the core temperature of the animals was monitored to ensure euthermia.

\section{Culture of fecal samples}

Fecal samples of antibiotic and vehicle group were collected on the 14th day. Similarly, FMT group fecal samples were collected on the 30th day. Each group fecal samples $(100 \mathrm{mg})$ were inoculated in $5 \mathrm{ml}$ broth medium and incubated $24 \mathrm{~h}$ under strict anaerobic conditions in constant temperature incubator at $37^{\circ} \mathrm{C}$ respectively. 


\section{Statistical analyses}

The number of repeats per group was provided in the legend and results were presented as mean \pm SEM . GraphPad Prism 7 (GraphPad software, San Diego, CA, USA) and R were used for statistical analysis of the data. The significance of the differences between two groups was analysed using unpaired Student's $t$ test, and multiple comparisons was performed by one-way analysis of variance (ANOVA) followed by Dunnett's post hoc test. All tests were two-tailed. Significant differences emerging from the above tests

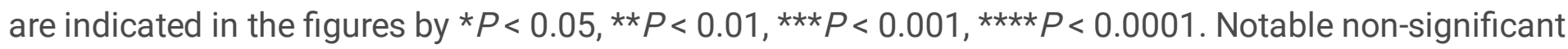
(and non-near significant) differences are indicated in the figures by "n.s". For all behavioral analyses, experimenters were blind to group assignment.

\section{Abbreviations}

GPR: G protein coupled receptor; TNF: Tumor necrosis factor; S1PR2: Sphingosine-1-phosphate receptor 2; CUS: Chronic unpredictable stress; PLS-DA: Partial least-square discriminant analysis; FMT: Fecal microbiome transplantation; AFMT: Anxiety fecal microbiome transplantation; CFMT: Control fecal microbiome transplantation; GF: Germ free; OFT: Open-field test; EPMT: Elevated plus maze test; FST: Forced swimming test; TST: Tail suspension test; SM: Sphingomyelin; PEs: Phosphatidylethanolamines; LysoPC: Lysophospholipid; CE: Cholesterol ester; AC: Adenylyl Cyclase; cAMP: Cyclic Adenosine 3',5'monophosphate; PKA: Protein kinase A.

\section{Declarations}

\section{Acknowledgements}

We thank the Laboratory Animal Science and Technology Center of Jiangxi University of traditional Chinese medicine for providing support for animal experiments

\section{Authors' contributions}

Baixi Shan, Yonggui Song and Dan Su designed, performed experiments, and wrote the manuscript. Sufen Zeng and Qiang Zeng helped with several experimental procedures. Zhou Liao, Tingting Wang , Jiagui Song and Chao Huang analyzed the metabolomics data.

\section{Funding}


This research was financially supported by National Natural Science Foundation of China (No.81860702), Scientific research project of Jiangxi University of Traditional Chinese Medicine (JXSYLXK-ZHYA0106, JXSYLXK-ZHYA0069, JXSYLXK-ZHYA0070), Jiangxi Province Nature Scientific Project (20171BAB205092, 20171BBG70112, 20171BCB23045).

\section{Ethics approval}

This study was approved by the approved by the Animal Care and Use Committee of Jiangxi University of Traditional Chinese Medicine (identification code: JZLLSC2018-0056).

\section{Availability of data and materials}

All data generated or analysed during this study are included in this published article and its supplementary information files or available from the corresponding author on reasonable request

\section{Consent for publication}

Not applicable.

\section{Competing interests}

The authors declare that they have no competing interests.

\section{References}

1. Shin LM, Liberzon I. The neurocircuitry of fear, stress, and anxiety disorders. Neuropsychopharmacolo. 2010;35:169.

2. Hammen C. Stress and . Annu Rev Clin Psychol. 2005;1:293-319.

3. Mayer EA, Tillisch K, Gupta A. Gut/brain axis and the microbiota. J Clin Invest. 2015;125:926-38.

4. Wong M, Inserra A, Lewis M, Mastronardi CA, Leong L, Choo J, et al. Inflammasome signaling affects anxiety and depressive-like behavior and gut microbiome composition. Mol Psychiatr. 2016;21:797.

5. Foster JA, Neufeld MV. Gut-brain axis: how the microbiome influences anxiety and : Trends in Neurosciences. Trends Neuroscie. 2013;36:305-12.

6. Gareau MG, Eytan W, Rodrigues DM, Joon Ho C, Whary MT, Philpott DJ, et al. Bacterial infection causes stress-induced memory dysfunction in mice. Gut. 2011;60:307-17. 
7. Desbonnet L, Clarke G, Shanahan F, Dinan TG, Cryan JF. Microbiota is essential for social development in the mouse. Mol Psychiatr. 2014;19:146-48.

8. Sampson T, Debelius J, Thron T, Janssen S, Shastri G, Ilhan ZE, et al. Gut Microbiota Regulate Motor Deficits and Neuroinflammation in a Model of Parkinson's Disease. Cell. 2016;167:1469.

9. Dickerson F, Severance E, Yolken R. The microbiome, immunity, and schizophrenia and bipolar disorder. Brain Behav Immun. 2017;62:46-52.

10. Rogers GB, Keating DJ, Young RL, Wong ML, Licinio J, Wesselingh S. From gut dysbiosis to altered brain function and mental illness: mechanisms and pathways. Mol Psychiatr. 2016;21.

11. Clarke G, Grenham S, Scully P, Fitzgerald P, Moloney R, Shanahan F, et al. The microbiome-gut-brain axis during early life regulates the hippocampal serotonergic system in a sex-dependent manner. Mol Psychiatr. 2013;18:666.

12. Cryan JF, Dinan TG. Mind-altering microorganisms: the impact of the gut microbiota on brain and behaviour. Nat Rev Neurosci. 2012;13:701-12.

13. Gerard C, Stilling RM, Kennedy PJ, Catherine S, Cryan JF, Dinan TG. Minireview: Gut microbiota: the neglected endocrine organ. Mol Endocrinol. 2014;28:1221-38.

14. Stilling RM, Wouw MVD, Clarke G, Stanton C, Tg TGD, Cryan JF. The neuropharmacology of butyrate: The bread and butter of the microbiota-gut-brain axis? Neurochem Int. 2016;99:110-32.

15. Rao SSC, Rehman A, Yu S, Andino NMD. Brain fogginess, gas and bloating: a link between SIBO, probiotics and metabolic acidosis. Clin Transl Gastroen. 2018;9:162-.

16. Hanstock TL, Mallet PE, Clayton EH. Increased plasma d-lactic acid associated with impaired memory in rats. Physiol Behav. 2010;101:653-59.

17. Bondi CO, Gustavo R, Gould GG, Alan F, Morilak DA. Chronic unpredictable stress induces a cognitive deficit and anxiety-like behavior in rats that is prevented by chronic antidepressant drug treatment. Neuropsychopharmacol. 2008;33:320.

18. Che Y, Zhou Z, Shu Y, Zhai C, Zhu Y, Gong S, et al. Chronic unpredictable stress impairs endogenous antioxidant defense in rat brain. Neurosci Lett. 2015;584:208-13.

19. Monteiro S, Roque S, De S-CD, Sousa N, Correia-Neves M, Cerqueira JJ. An efficient chronic unpredictable stress protocol to induce stress-related responses in C57BL/6 mice. Front Psychiatry. 2015;6:6.

20. Zheng P, Zeng B, Zhou C, Liu M, Fang Z, Xu X, et al. Gut microbiome remodeling induces depressivelike behaviors through a pathway mediated by the host's metabolism. Mol Psychiatr. 2016;21:786-96.

21. Yue N, Li B, Yang L, Han QQ, Huang HJ, Wang YL, et al. Electro-Acupuncture Alleviates Chronic Unpredictable Stress-Induced Depressive- and Anxiety-Like Behavior and Hippocampal Neuroinflammation in Rat Model of . Front Mol Neurosci. 2018;11:149.

22. Xu J, Chen L, Su J, Liu Z, Jie C, Lin Q, et al. The anxiolytic-like effects of ginsenoside Rg3 on chronic unpredictable stress in rats. Sci Rep-UK. 2018;8. 
23. Zhou T, Zhu H, Fan Z, Wang F, Chen $Y$, Liang H, et al. History of winning remodels thalamo-PFC circuit to reinforce social dominance. Science. 2017;357:162-68.

24. Song Y, Shan B, Li H, Feng B, Peng H, Jin C, et al. Safety investigation of Pulsatilla chinensis saponins from chronic metabonomic study of serum biomedical changes in oral treated rat. $\mathrm{J}$ Ethnopharmacol. 2019;235:435-45.

25. Zeng Q, Liu Y, Song Y, Feng B, Xu P, Shan B, et al. A UHPLC-MS/MS method coupled with simple and efficient alkaline hydrolysis for free and total determination of conjugate nanomedicine: Pharmacokinetic and biodistribution study of poly (L-glutamic acid)-graft-methoxy poly (ethylene glycol)/combretastatin A4. J. Pharm. Biomed. Anal., 2019, 169: 215-224.

26. Liu K, Song Y, Liu Y, Peng M, Li H, Li X, et al. An integrated strategy using UPLC-QTOF-MSE and UPLCQTOF-MRM (enhanced target) for pharmacokinetics study of wine processed Schisandra Chinensis fructus in rats. J. Pharm. Biomed. Anal., 2017, 139: 165-178.

27. Olson CA, Vuong HE, Yano JM, Liang QY, Nusbaum DJ, Hsiao EY. The Gut Microbiota Mediates the Anti-Seizure Effects of the Ketogenic Diet. Cell. 2018;173:497.

28. Koh A, Molinaro A, Ståhlman M, Khan MT, Schmidt C, Mannerås-Holm L, et al. Microbially produced imidazole propionate impairs insulin signaling through mTORC1. Cell. 2018;175:947-61. e17.

29. Yumi Y, Takeshi N, Akihiro K, Ryutaro I, Tetsuya K, Masutaka F. Radiation-induced skin ulcer and rib fractures following percutaneous coronary intervention (PCI): A case of right back skin ulcer and adjacent rib fractures after single PCl. J Dermatol. 2015;42:508-10.

30. Wakita H, Tomimoto H, Akiguchi I, Matsuo A, Lin JX, Ihara M, et al. Axonal damage and demyelination in the white matter after chronic cerebral hypoperfusion in the rat. Brain Res. 2002;924:63-70.

31. Ryo O, Hidekazu T, Tadakazu K, Hideaki W, Ichiro A, Hiroshi S, et al. Upregulation of ceramide and its regulating mechanism in a rat model of chronic cerebral ischemia. Brain Res. 2004;1023:31-40.

32. Zhu F, Guo R, Wang W, Ju Y, Wang Q, Ma Q, et al. Transplantation of microbiota from drug-free patients with schizophrenia causes schizophrenia-like abnormal behaviors and dysregulated kynurenine metabolism in mice. Mol Psychiatr. 2019:1-14.

33. Kang D-W, Adams JB, Gregory AC, Borody T, Chittick L, Fasano A, et al. Microbiota Transfer Therapy alters gut ecosystem and improves gastrointestinal and autism symptoms: an open-label study. Microbiome. 2017;5:10.

34. Cai TQ, Ning R, Lan J, Kang C, Kash S, Chen R, et al. Role of GPR81 in lactate-mediated reduction of adipose lipolysis. Biochem Biophys Res Commun. 2008;377:987-91.

35. Husted AS, Trauelsen M, Rudenko O, Hjorth SA, Schwartz TW. GPCR-Mediated Signaling of Metabolites. Cell Metab. 2017;25:777-96.

36. Kreisberg RA. Lactate homeostasis and lactic acidosis. Ann Intern Med. 1980;92:227.

37. Pruett ED. FFA mobilization during and after prolonged severe muscular work in men. J Appl Physiol. 1970;29:809. 
38. Ahmed K, Tunaru S, Tang C, Müller M, Gille A, Sassmann A, et al. An autocrine lactate loop mediates insulin-dependent inhibition of lipolysis through GPR81. Cell metab. 2010;11:311-19.

39. Zheng P, Zeng B, Liu M, Chen J, Pan J, Han Y, et al. The gut microbiome from patients with schizophrenia modulates the glutamate-glutamine-GABA cycle and schizophrenia-relevant behaviors in mice. Sci Adv. 2019;5:eaau8317.

40. Pearson-Leary J, Zhao C, Bittinger K, Eacret D, Luz S, Vigderman AS, et al. The gut microbiome regulates the increases in depressive-type behaviors and in inflammatory processes in the ventral hippocampus of stress vulnerable rats. Mol Psychiatr. 2019:1.

41. Goyal G, Zheng J, Adam E, Steffes G, Jain M, Klavins K, et al. Sphingolipid-dependent Dscam sorting regulates axon segregation. Nat Commun. 2019;10:813.

42. Uribarri J, Oh MS, Carroll HJ. D-lactic acidosis. A review of clinical presentation, biochemical features, and pathophysiologic mechanisms. Medicine. 1998;77:73.

43. Petersen C. D-Lactic Acidosis. Nutr Clin Pract. 2005;20:634.

44. Ahmed K, Tunaru S, Offermanns S. GPR109A, GPR109B and GPR81, a family of hydroxy-carboxylic acid receptors. Trends Pharmacol Sci. 2009;30:557-62.

45. Carlson LA. Studies on the effect of nicotinic acid on catecholamine stimulated lipolysis in adipose tissue in vitro. J Intern Med. 2010;173:719-22.

46. Takatoshi S, Masazumi K, Jun T, Shun-Ichiro M, Tetsu S, Takahide O, et al. Molecular identification of nicotinic acid receptor. Biochem Bioph Res Co.2003;303:364-69.

47. Tunaru S, Kero JA, Wufka C, Blaukat A, Pfeffer K, Offermanns S. PUMA-G and HM74 are receptors for nicotinic acid and mediate its anti-lipolytic effect. Nat Med. 2003;9:352-55.

\section{Figures}



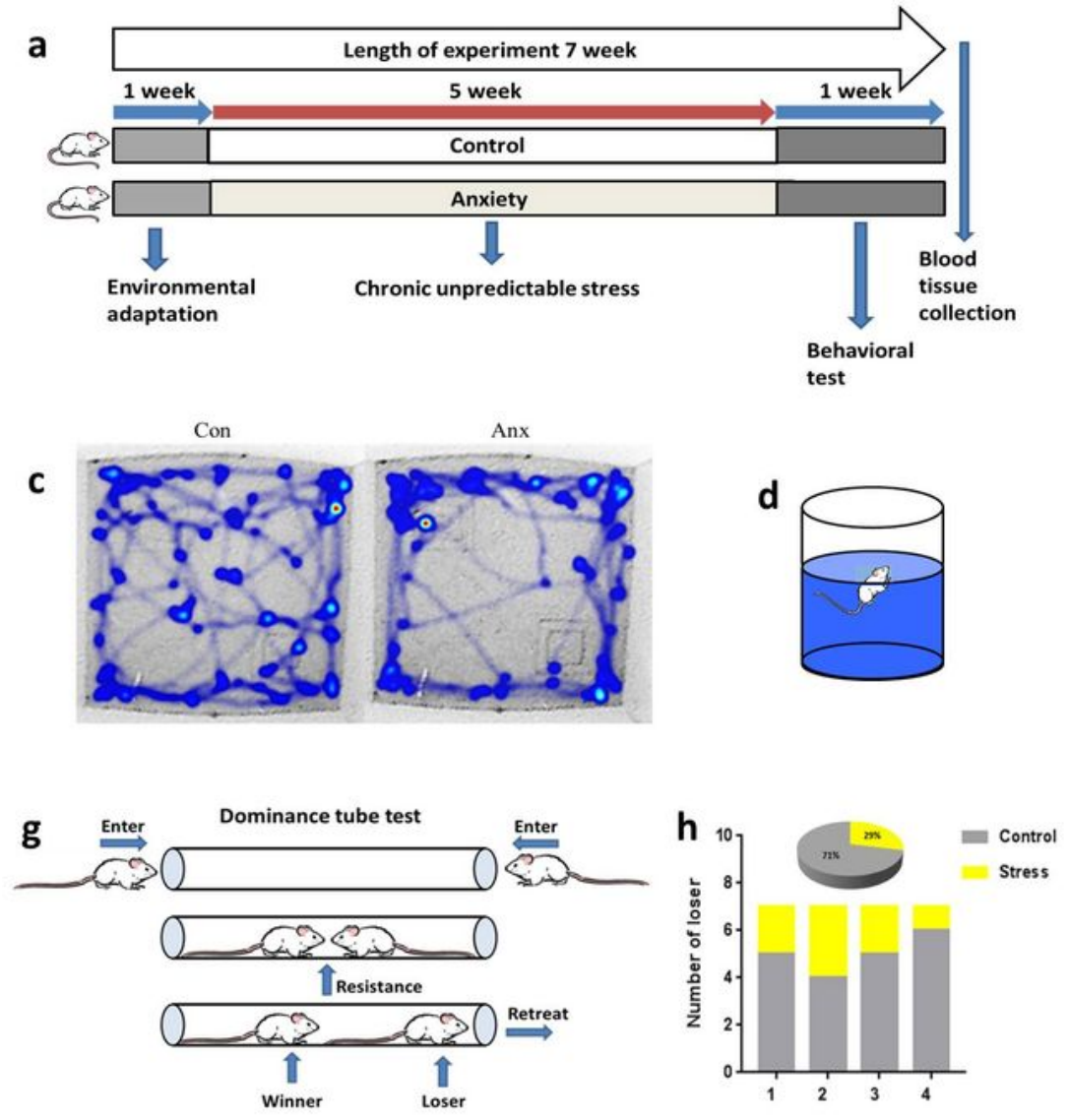

b
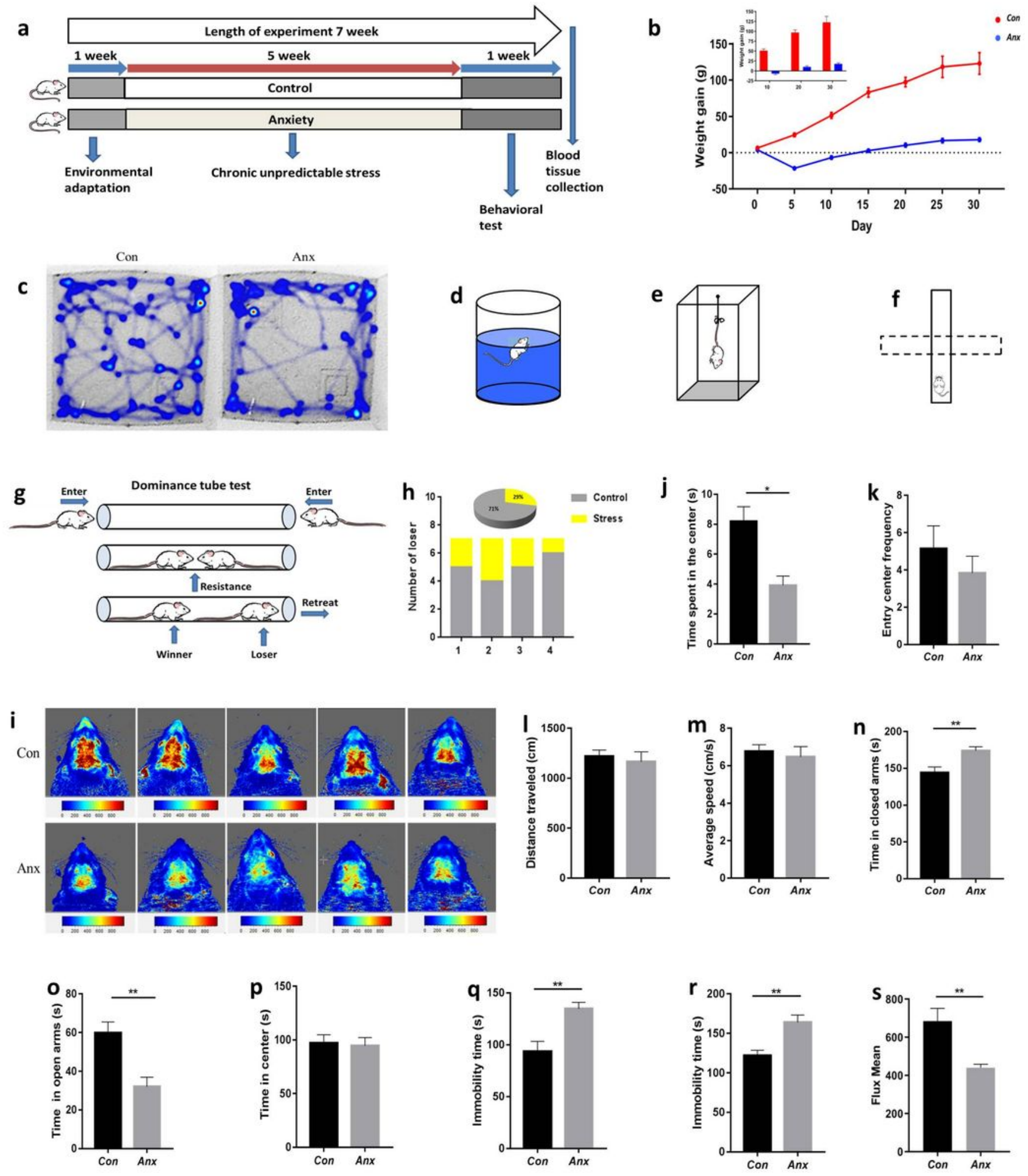

\section{Figure 1}

Behavioral comparisons between the anxiety group and the control group. a Schematic diagram of experimental procedure during the 7 weeks. b Weight gain during 35 days of chronic unpredictable stress group and normal group. c Open-field test (OFT). d Forced swimming test (FST). e Tail suspension test (TST). $f$ Elevated plus maze test (EPMT). $g$ and $h$ Tube test (TT) ( $n=10-15$ per group). i Cerebral blood flow. j-m Open-field test. Compared to the control group rats, time spend in the center and entry center 
frequency were reduced in the anxiety group rats ( $n=10-15$ per group). $n-p$ Elevated plus maze test. Compared to the control group rats, time spend in closed arms was significantly increased, and time in open arms was significantly reduced in the anxiety group rats ( $n=10-15$ per group). $q$ In the FST, the immobility time of anxious rats significantly increased ( $n=10-15$ per group). $r$ In the TST, the immobility time of anxious rats significantly increased ( $n=10-15$ per group). $s$ Laser Doppler imaging of brain showed that rats in anxiety group were accompanied by cerebral ischemia. Data are mean \pm SEM. $* P<$ $0.05,{ }^{*} \mathrm{P}<0.01$. Unpaired two-tailed Student's t tests.
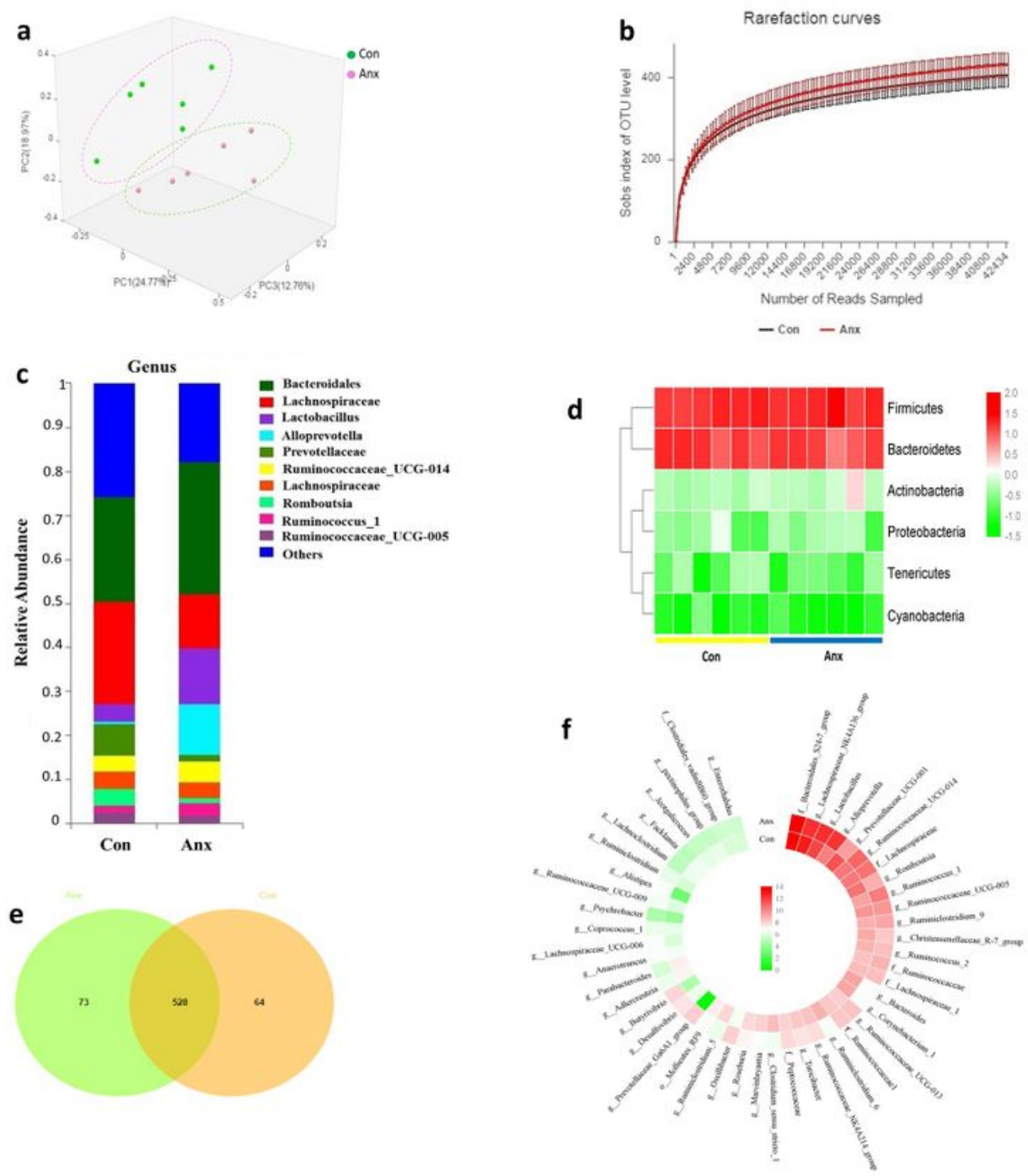

g

Wilcoxon rank-sum test bar plot on Genus level
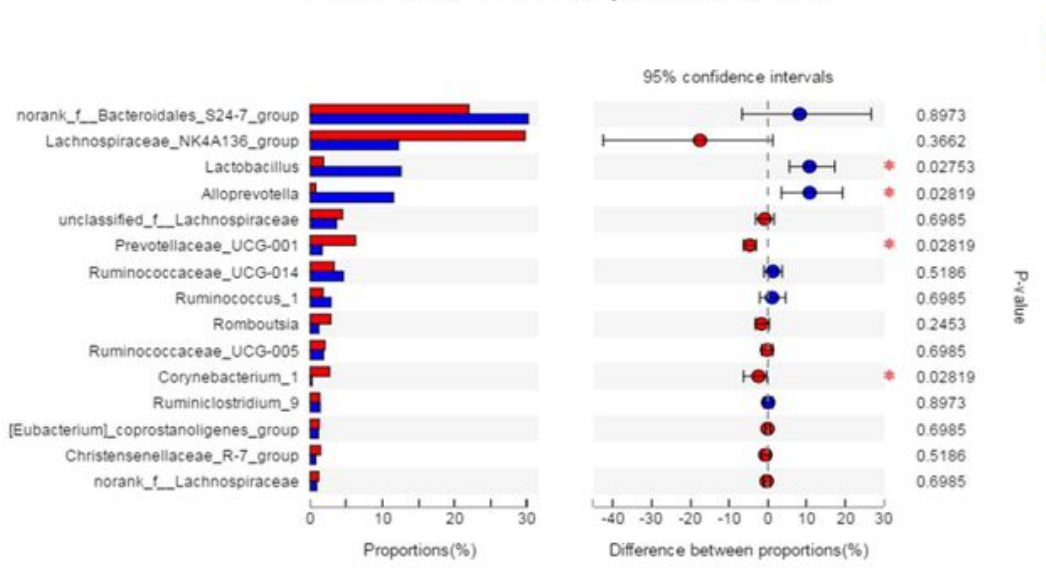


\section{Figure 2}

16S rRNA gene sequencing reveals changes to microbial diversity in anxiety group. a Three-dimensional principal coordinate analysis (PCoA) of unweighted UniFrac distances showed an obvious difference in gut microbiotic composition between anxiety and control group ( $n=6 /$ anxiety group, violet plots; $n=6 /$ control group, green plots). The percentage of variation explained by principal coordinates is marked on the axes. b Rarefaction curves depicted from randomly subsampled data sets with the same number of $16 \mathrm{~S}$ sequences. The near saturated rarefaction curve indicates that the vastness of microbial diversity was retrieved from each sample. c Taxonomic distributions of bacteria from colonic luminal content $16 \mathrm{~S}$ rRNA sequencing data at the genus levels between the anxiety group and control group. $d$ Heatmap of microbial phylum level abundance (top 6). e Venn diagram of bacterial species number in feces of anxiety group and control group. f Heatmap of microbial genus level abundance (top 50). g Phylotypes significantly different between anxiety and control groups at genus level. Statistical analysis was performed by the Wilcoxon rank-sum test. $n=6 /$ group. * $P<0.05$. 


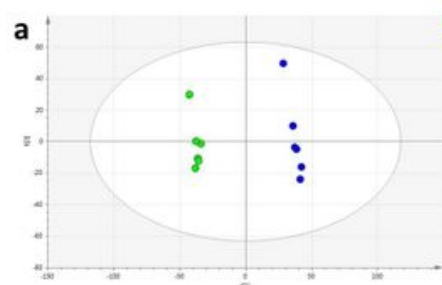

$\because \mathrm{b}$
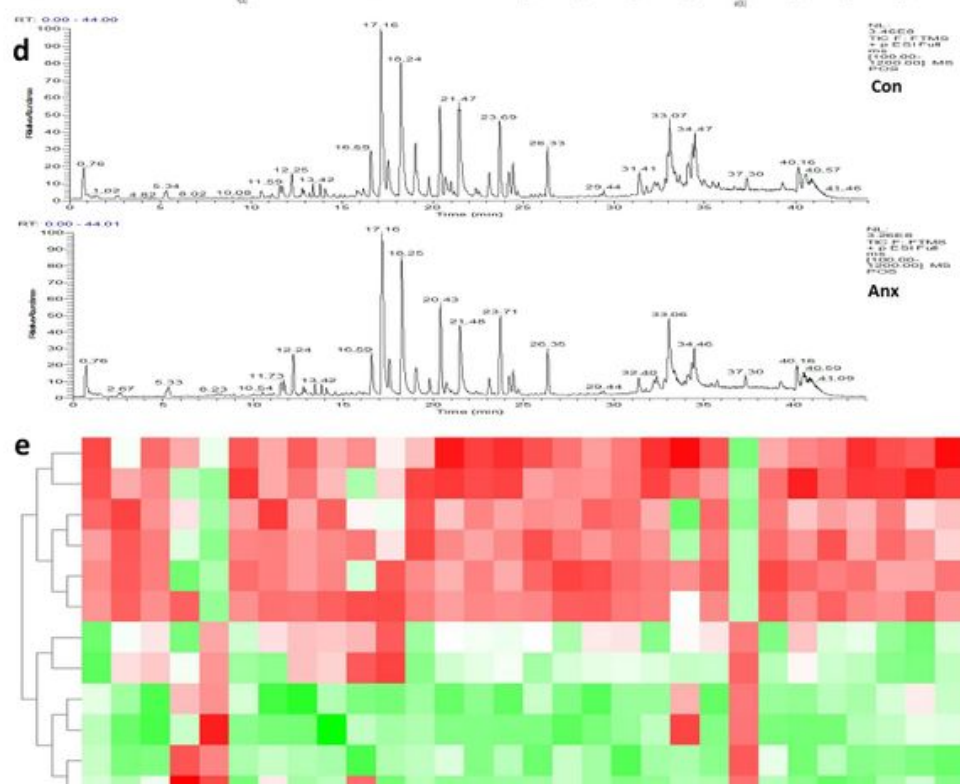

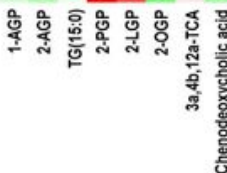
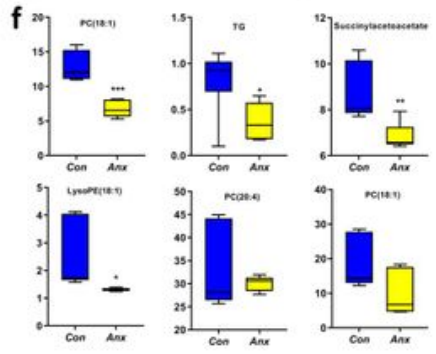

con Ans
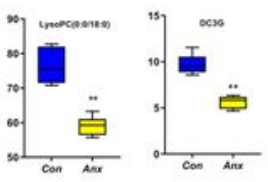

帛
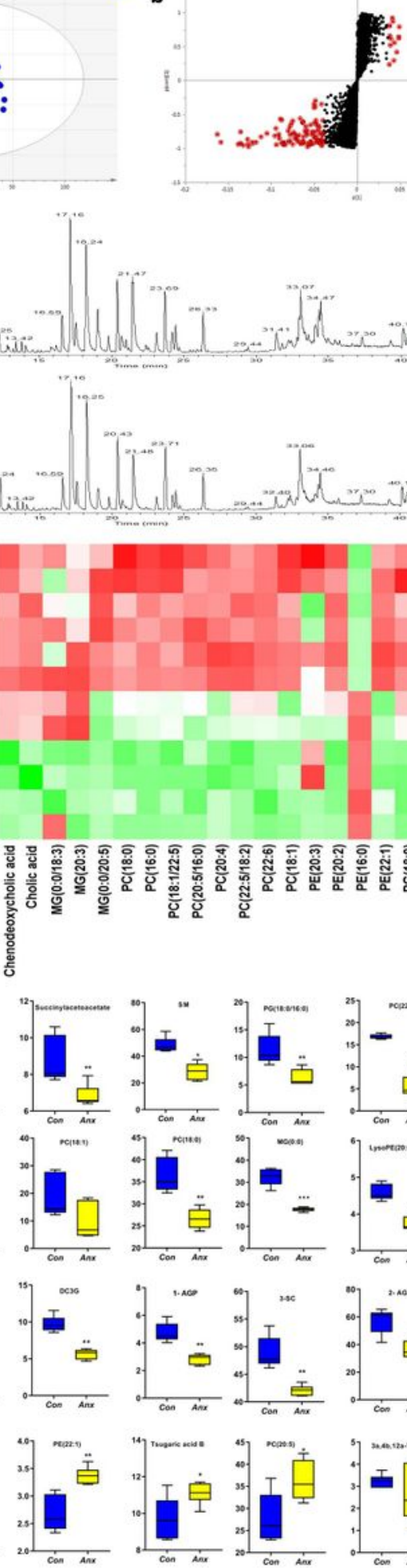

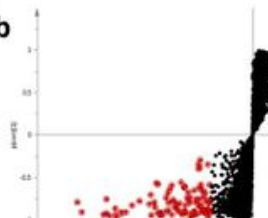

?: $\therefore$

c

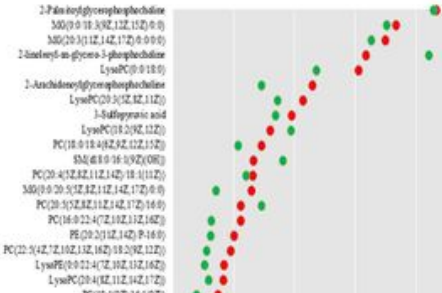

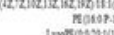

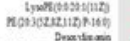

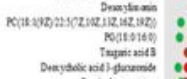

5

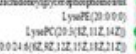

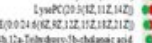

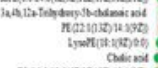
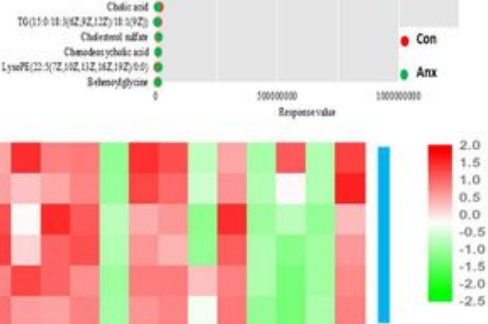

Con

Anx

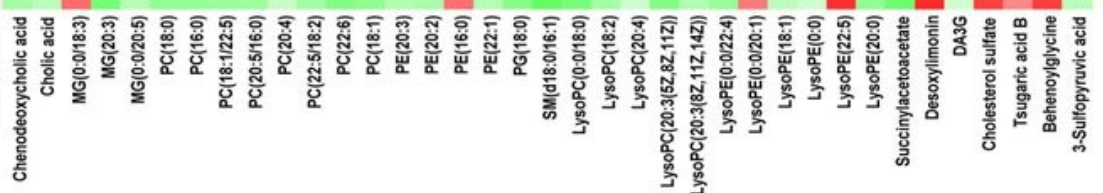


scatter plot of rats in anxiety group and control group. The X-axis represents the response value. $d$ Typical base peak intensity (BPI) chromatograms show differences in rat plasma metabolites between anxiety group and control group. e Heatmap analysis of 45 significant difference metabolites from the anxiety group and control group rats. Red and green highlights indicate increased and decreased metabolites in the anxiety group compared to the control group rats. $f$ Relative intensities of individual metabolites of phospholipids, lysophosphatides, cholesterol, fatty acids and other class metabolites in the anxiety and control group rats. The vertical axis is the relative intensity of individual metabolites.TG triglycerides, SM sphingomyelin, MG monoacylglyceride, PC phosphatidylcholine, PE phosphatidylethanolamine, LysoPC lysophospholipid, LysoPE lysophosphatidylethanolamine, AGP Arachidonoylglycerophosphoinositol, PGP Palmitoylglycerophosphocholine, LGP linoleoyl-sn-glycero-3-phosphocholine, OGP Oleoylglycerophosphocholine, TCA Trihydroxy-5b-cholanoic acid, DA3G Deoxycholic acid 3-glucuronide. Data are presented as means \pm SEM. Student's t test was used for the significance of difference between

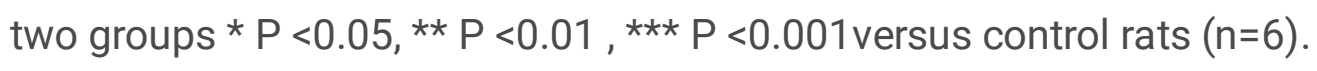


a
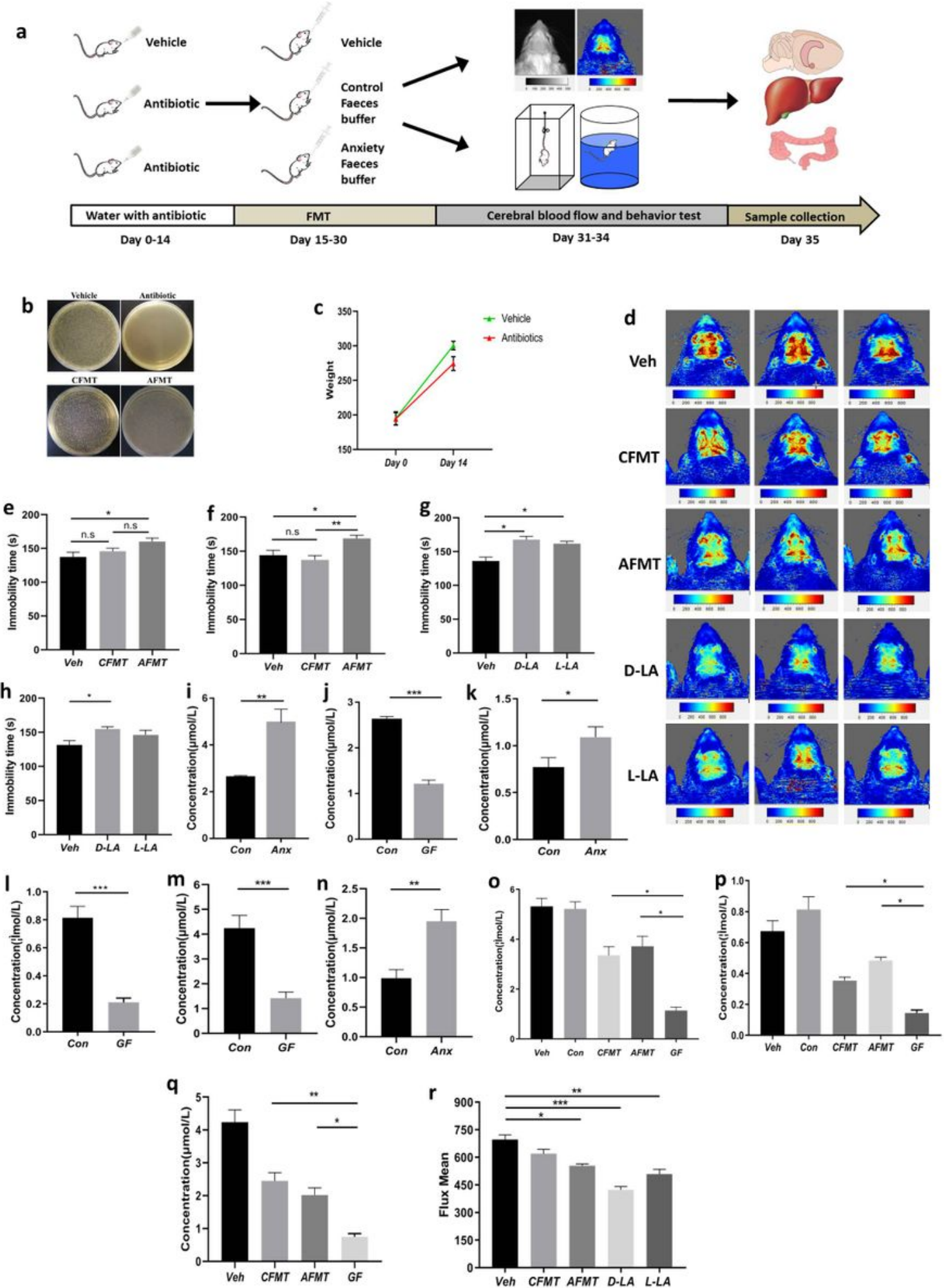

\section{Figure 4}

Effects of fecal microbiota transplantation on behavior, brain blood flow and lactic acid concentration in rat. a Experimental design. $b$ Culture of fecal microorganisms in vitro and fecal transplants on reshaping the gut microbiome. $c$ Weight changes of rats treated with oral antibiotics and PBS for 14 days. $d$ Cerebral blood flow. e and $\mathrm{h}$ In the TST, immobility time was significantly higher in rats treated with 'anxiety microbiota' (e) and L-lactic acid ( $g$ ) relative to vehicle groups ( $\mathrm{n}=8-10$ per group). $\mathrm{f}$ and $\mathrm{g}$ The 
'anxiety microbiota' recipients and lactic acid displayed an increased duration of immobility in the FST compared to 'healthy microbiota' recipients and vehicle ( $n=8-10$ per group). i, j and o Concentration of Llactic acid in plasma. $\mathrm{k}, \mathrm{I}$ and $\mathrm{p}$ Concentration of D-lactic acid in plasma. $\mathrm{m}, \mathrm{n}$ and $\mathrm{q}$ Concentration of Llactic acid in fecal. $r$ Effects of fecal microbiota transplantation and lactic acid on cerebral blood flow in rats. Data are presented as means \pm SEM. Student's $t$ test was used for the significance of difference between two groups, one-way ANOVA followed by Dunnett's post hoc test for multiple comparisons was used for three or more groups, * $\mathrm{P}<0.05$, ** $\mathrm{P}<0.01$, *** $\mathrm{P}<0.001$.
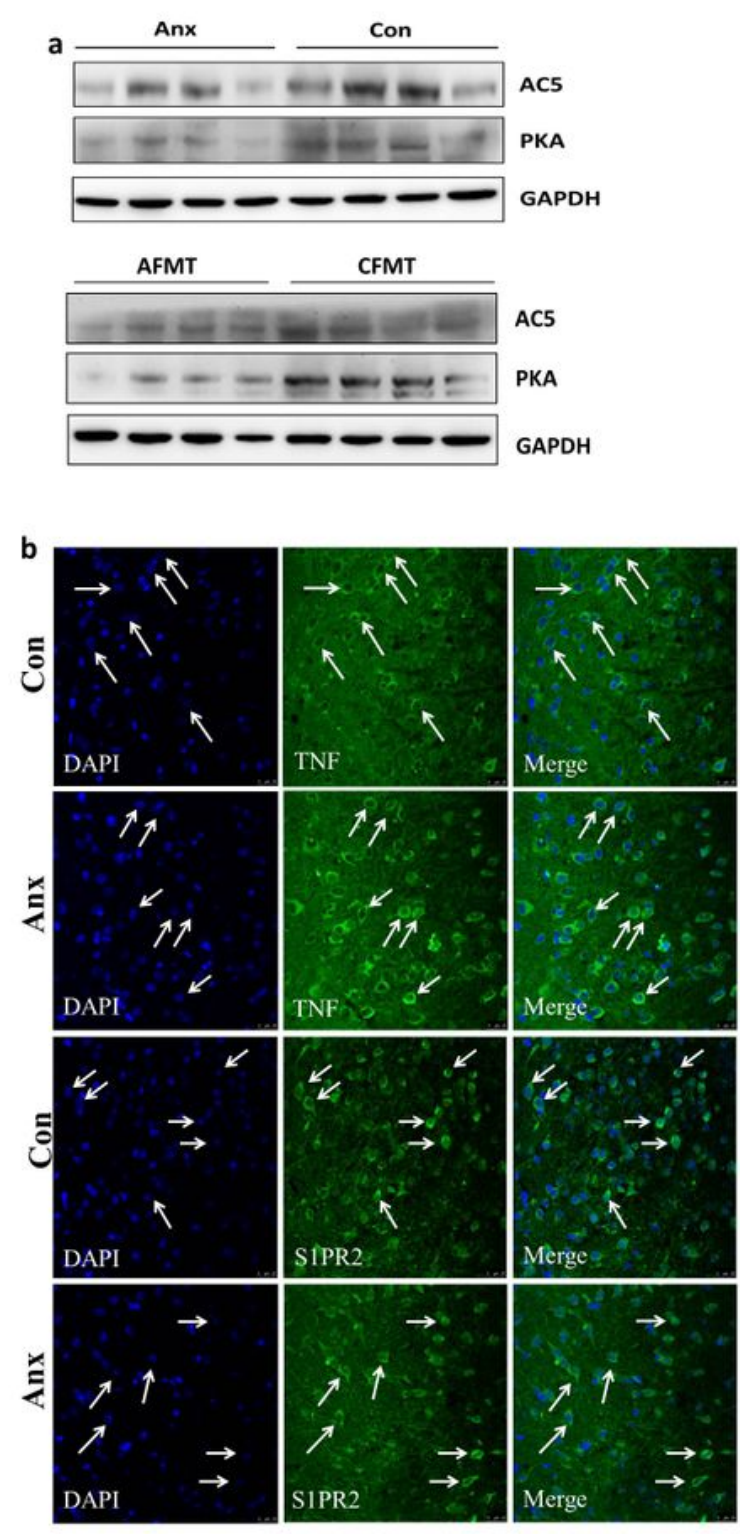
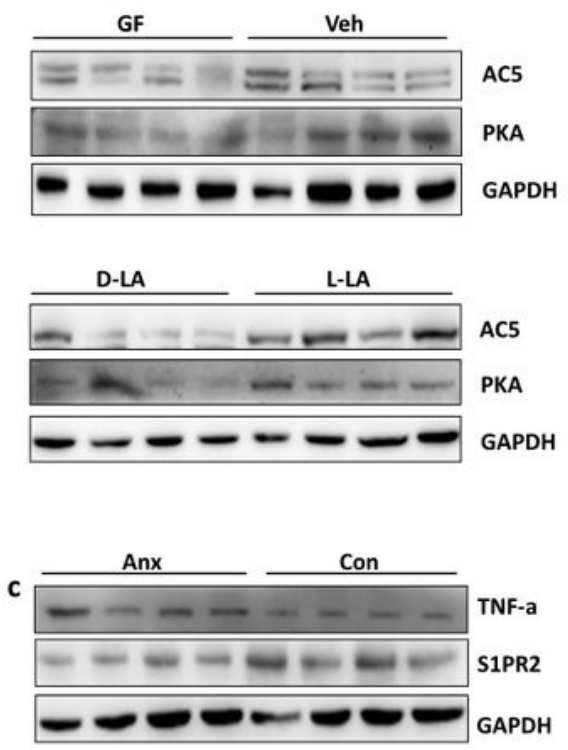

GPR81
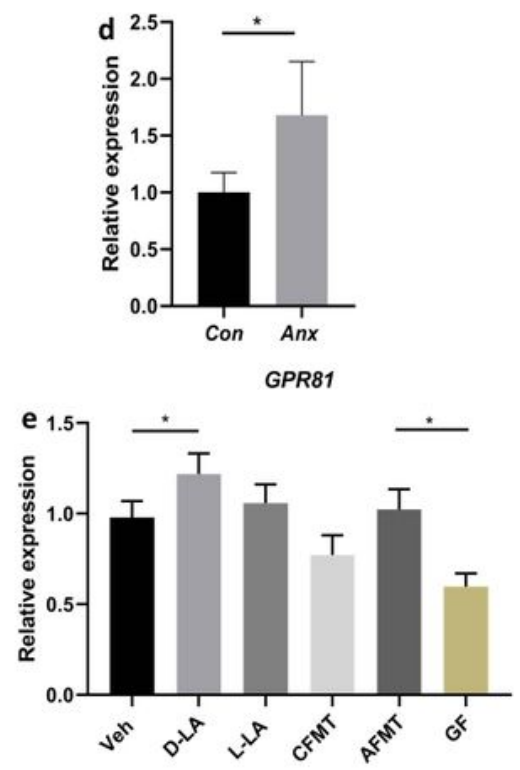
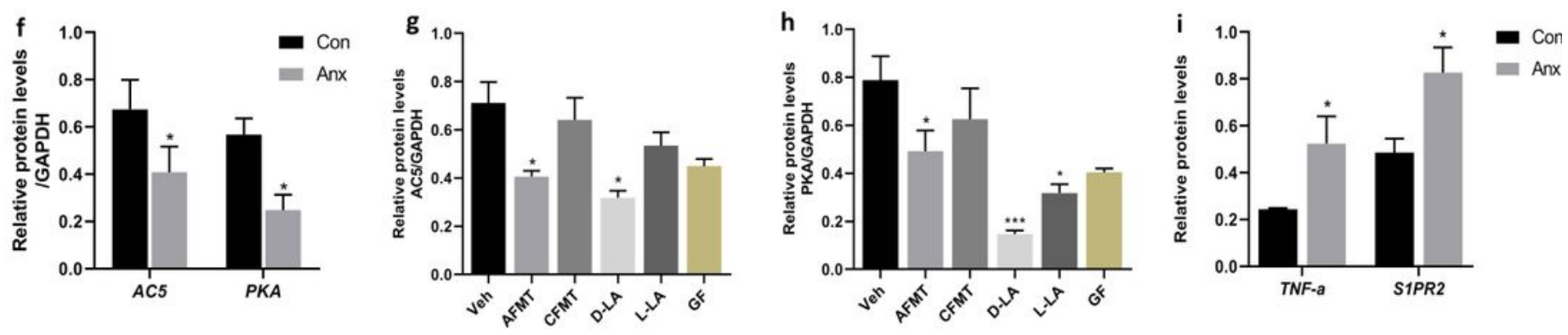


\section{Figure 5}

Anxiety disorder correlates with inhibition of AC5 and PKA in liver and expression of inflammatory cytokines in brain. a $\mathrm{g} \mathrm{h}$ The protein expression and relative quantitative data of AC5 and PKA in Anx, Con, GF, Veh, AFMT, CFMT, L-LA, D-LA. b Representative immunofluorescent staining of TNF-a and S1PR2 in brain. $\mathrm{c}$ i The protein expression and relative quantitative data of TNF-a and S1PR2 in Anx and Con. d e Relative expression of GPR81 mRNA in liver. Data are presented as means \pm SEM. Student's t test was used for the significance of difference between two groups, one-way ANOVA followed by Dunnett's post hoc test for multiple comparisons was used for three or more groups, $* P<0.05, * \star P<0.01, * \star \star P<0.001$.

\section{Supplementary Files}

This is a list of supplementary files associated with this preprint. Click to download.

- TableS1.xlsx

- SupplementaryInformation.docx 